\title{
Thermal and hard scales in transverse momentum distributions, fluctuations, and entanglement
}

\author{
X. Feal $\odot,{ }^{1,2}$ C. Pajares $\odot,{ }^{2}$ and R. A. Vazquez $\odot^{2}$ \\ ${ }^{1}$ Physics Department, Brookhaven National Laboratory, Bldg. 510A, Upton, New York 11973, USA \\ ${ }^{2}$ IGFAE/Instituto Galego de Física de Altas Enerxías \& Universidade de Santiago de Compostela, 15782 Santiago, Spain
}

(Received 31 December 2020; revised 3 March 2021; accepted 16 September 2021; published 7 October 2021)

\begin{abstract}
We analyze the transverse momentum distributions of $p p, p \mathrm{~Pb}, \mathrm{XeXe}$, and $\mathrm{PbPb}$ collisions at different RHIC and LHC energies and centralities as well as the corresponding distributions for Higgs production decaying into $\gamma \gamma$ and $4 l$. A simple linear relation is found between the effective thermal temperature and the hard scale, approximately valid for all processes and mainly determined by the hard scale fluctuations. To go further, it is shown that the whole spectrum of $p p$ collisions can be described by a single function showing that the thermal temperature is determined solely by the hard scale and its fluctuations. The possible relation between the multiplicities of the soft and hard scales is explored.
\end{abstract}

DOI: 10.1103/PhysRevC.104.044904

\section{INTRODUCTION}

The apparent thermal features and the collective behavior observed in proton-proton collisions has challenged our current understanding of the small collision systems, where the application of the conventional hydrodynamical explanation seems to be questionable [1,2]. On the other hand, theoretical studies of quenches in entangled systems described by $(1+1)$-dimensional conformal field theories of expanding quantum fields and strings, have shown that these systems behave as a generalized Gibbs ensemble with an effective temperature set by the energy cutoff for the ultraviolet modes [3-6]. In the last years there has been a large activity in the field [7-18].

In a high-energy collision a hard parton interaction produces a rapid quench of the entangled partonic state [19] and thus the characteristic effective temperature-inferred from the exponential shape of the transverse momentum distribution (TMD) of the secondary particles produced in the collision-can depend on the energy scale of the hard process, which works as an ultraviolet cutoff of the quantum modes resolved by the collision. This possibility has been recently studied in charged particle and Higgs boson production in $p p$ and $\mathrm{PbPb}$ collisions at very different energies and multiplicities, where a relation between the hard scale and the effective temperature has been found [20-22]. In this paper we extend our previous analysis and perform an extensive study of the energy and multiplicity dependence of the hard and soft scales in $p p$ collisions [23-29] as well as $p \mathrm{~Pb}$ [30], XeXe [31], and $\mathrm{PbPb}$ [26] collisions. We show that the relation between

Published by the American Physical Society under the terms of the Creative Commons Attribution 4.0 International license. Further distribution of this work must maintain attribution to the author(s) and the published article's title, journal citation, and DOI. Funded by SCOAP . both scales is determined approximately by the inverse of the normalized fluctuations of the number of partons of the initial wave function or, equivalently, of the normalized fluctuations of the hard scale.

In the entanglement picture of the nucleon as the underlying mechanism for the fast thermalization of partons, a hard process, with transverse momentum $p_{\perp}$, probes only the region of the space $H$ of transverse size $1 / p_{\perp}$. Let us denote by $S$ the region of space complementary to $H$. The initial state is described by the wave function

$$
|\Psi\rangle=\sum_{n} \alpha_{n}\left|\Psi_{n}^{H}\right\rangle \otimes\left|\Psi_{n}^{S}\right\rangle
$$

of a suitably chosen orthonormal set of states $\left|\Psi_{n}^{H}\right\rangle$ and $\left|\Psi_{n}^{s}\right\rangle$ localized in the domains $H$ and $S$, with different numbers $n$ of partons. The state (1) cannot be separated into a product $\left|\Psi^{H}\right\rangle \otimes\left|\Psi^{S}\right\rangle$, and therefore $|\Psi\rangle$ is entangled. The density matrix of the mixed state probed in the region $H$ is

$$
\rho_{H}=\operatorname{Tr}_{S} \rho=\sum_{n}\left\langle\Psi_{n}^{S} \mid \Psi\right\rangle\left\langle\Psi \mid \Psi_{n}^{S}\right\rangle=\sum_{n}\left|\alpha_{n}\right|^{2}\left|\Psi_{n}^{H}\right\rangle\left\langle\Psi_{n}^{H}\right|,
$$

where $\left|\alpha_{n}\right|^{2} \equiv p_{n}$ is the probability of having a state with $n$ partons. We can consider that a high-momentum partonic configuration of the initial state when the interaction takes place undergoes a rapid quench. The onset $\tau$ of this hard interaction is given by the hardness scale, $\tau \sim 1 / p_{\perp}$. Because $\tau$ is small, the quench creates a highly excited multiparticle state. The produced particles have a thermal-like exponential spectrum with an effective temperature which is determined by the hard scale and the fluctuations on the number of partons. With these considerations, we fit the different TMDs by an exponential distribution and a power-like distribution [20,21,32]

$$
\frac{1}{N_{e v}} \frac{1}{2 \pi p_{\perp}} \frac{d^{2} N_{e v}}{d \eta d p_{\perp}}=A_{t h} e^{-m_{\perp} / T_{t h}}+\frac{A_{h}}{\left(1+m_{\perp}^{2} / k T_{h}^{2}\right)^{k}},
$$




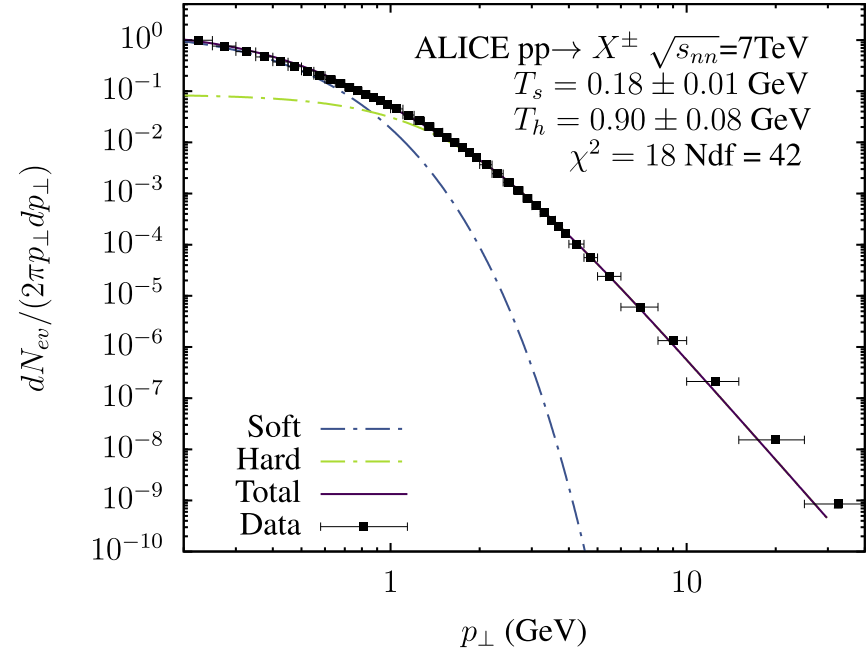

FIG. 1. Charged particle TMD in $p p$ collisions at $\sqrt{s_{n n}}=7 \mathrm{TeV}$ in the centrality class $0 \%-5 \%$. Fit to (3) in the interval $0.17<p_{\perp}<$ $32.5 \mathrm{GeV} / c$. Normalized data taken from Ref. [27] with systematic and statistical uncertainties combined. Unidentified charged particle mass set to $m_{X}=0.23 \mathrm{GeV} / c^{2}$, corresponding to the weighted average mass of the $\pi^{ \pm}, K^{ \pm}$, and $p$ channels.

where $T_{t h}$ is the effective temperature, $T_{h}$ is the hard scale, and $k$ a parameter that characterizes the perturbative falloff of the distribution at high $p_{\perp}$.

In the next sections, we show that the relation between the two scales is given by the size of the fluctuations of the hard scale. This relation is universal, valid for $p p, p \mathrm{~Pb}, \mathrm{XeXe}$, and $\mathrm{PbPb}$ for all energies and centralities. In the case of $p p$ collisions, we are able to describe the whole $p_{\perp}$ spectrum with a single function for energies ranging from the BNL Relativistic Heavy Ion Collider (RHIC) to the LHC. This function provides us with the low- $p_{\perp}$ fluctuations needed to improve the universal agreement found in $A A$ and $p A$ collisions.

\section{THE THERMAL TEMPERATURE DETERMINED BY THE HARD SCALE AND ITS FLUCTUATIONS}

A fit to (3) of the TMD of charged particles measured by the ALICE Collaboration [27] in the most central $p p$ collisions at a center-of-mass energy of $\sqrt{s_{n n}}=7 \mathrm{TeV}$ is shown in Fig. 1. In Fig. 2 we show the $p \mathrm{~Pb}$ scenario, in which the TMD of $\pi^{ \pm}$in collisions at $\sqrt{s_{n n}}=5.02 \mathrm{TeV}$ is shown, and in Fig. 3 the TMD of charged particles in $\mathrm{PbPb}$ collisions is shown at the same center-of-mass energy. The general behavior of these TMD in all the analyzed cases consists in a soft thermal contribution with a characteristic temperature $T_{t h} \in 0.15-0.20 \mathrm{GeV}$, representing typically the $\approx 80 \%-90 \%$ of the total inclusive cross section, plus a hard tail dominant at high energies, with temperature $T_{h} \in 0.7-1 \mathrm{GeV}$ and falloff $k \in 3-5$, exhibiting the characteristic features of the perturbative limit. The major source of uncertainty in the determination of these three parameters by fits to experimental data are the systematic uncertainties, the large bins usually required to collect very-high- $p_{\perp}$ events and the signals of final-state interactions in large colliding systems. The results

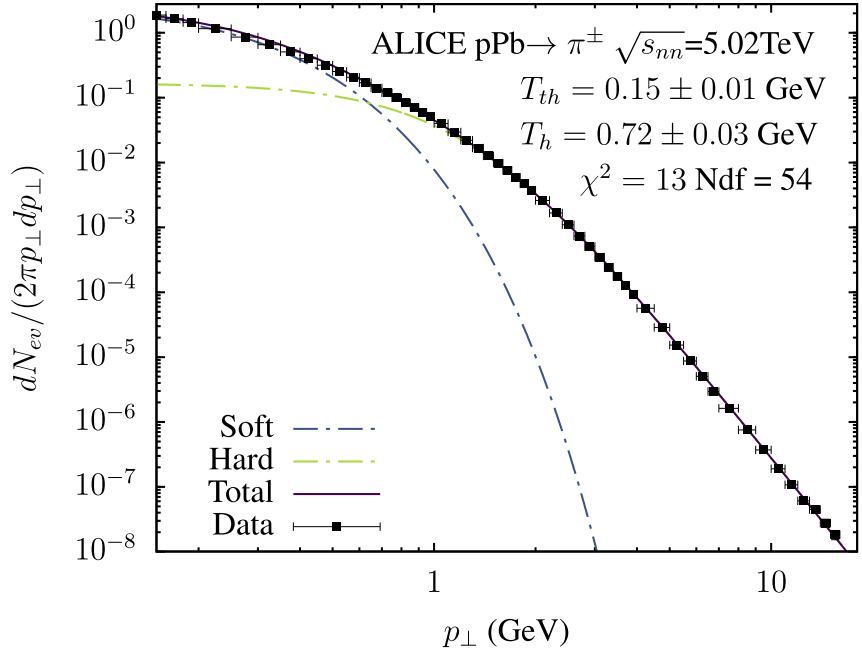

FIG. 2. $\pi^{ \pm}$TMD in $p \mathrm{~Pb}$ collisions at $\sqrt{s_{n n}}=5.02 \mathrm{TeV}$ in the centrality class $0 \%-5 \%$. Fit to (3) in the interval $0.1<p_{\perp}<$ $19 \mathrm{GeV} / c$. Normalized data taken from Ref. [30] with systematic and statistical uncertainties combined. Pion mass set to $m_{\pi}=$ $0.14 \mathrm{GeV} / c^{2}$.

of the rest of the fits of the TMDs to (3) are shown in tables, with the list of extracted quantities and uncertainties and the fit parameters disclosed, and depicted also in the accompanying figures. Systematic uncertainties are included in the fits as random errors, thus the obtained $\chi^{2} / \mathrm{Ndf}$ values are generally large and the fit parameter errors are overestimated.

In Table I, we show the values of $T_{t h}, T_{h}$, and $k$ obtained from the fits to the TMD of charged particles produced in $p p$ collisions at very different RHIC and LHC energies. In Table II, we show the results from the fits to the TMD of charged pions for several multiplicity bins in $p p$ collisions at

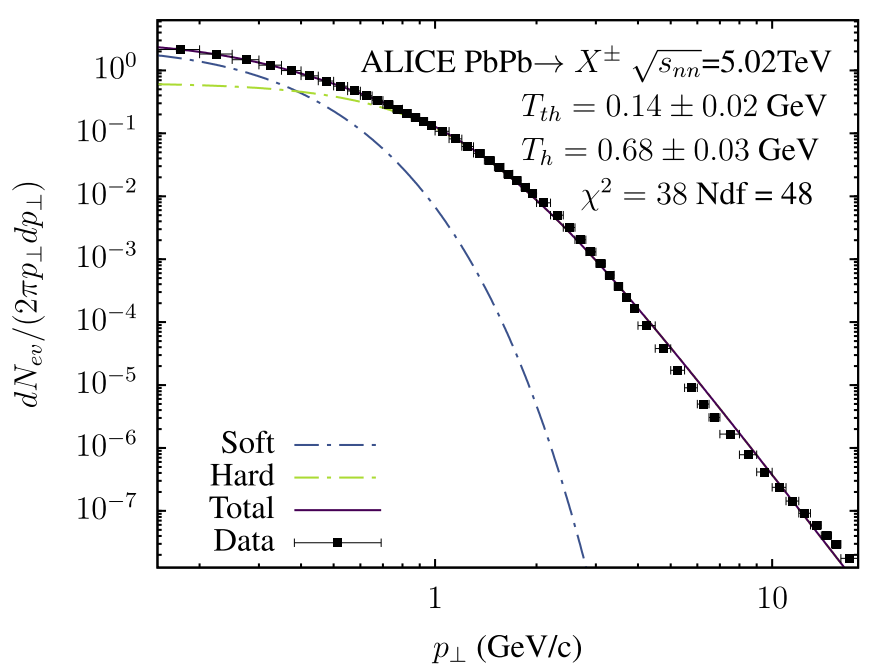

FIG. 3. Charged particle TMD in $\mathrm{PbPb}$ collisions at $\sqrt{s_{n n}}=$ $5.02 \mathrm{TeV}$ in the centrality class $0 \%-5 \%$. Fit to (3) in the interval $0.2<p_{\perp}<17 \mathrm{GeV} / c$. Normalized data taken from Ref. [26] with systematic and statistical uncertainties combined. Unidentified charged particle mass set to $m_{X}=0.23 \mathrm{GeV} / c^{2}$, corresponding to the weighted average mass of the $\pi^{ \pm}, K^{ \pm}$, and $p$ channels. 
TABLE I. Temperatures, falloff $k$, and fit parameters of the TMD of charged particles in $p p$ collisions at different RHIC and LHC colliding energies, with data taken from Refs. [23-29].

\begin{tabular}{lccccc}
\hline \hline$\sqrt{s_{n n}}$ & $T_{t h}(\mathrm{GeV})$ & $T_{h}(\mathrm{GeV})$ & $k$ & $\chi^{2}$ & Ndf \\
\hline $64 \mathrm{GeV}$ & $0.15 \pm 0.07$ & $0.68 \pm 0.16$ & $5.5 \pm 0.1$ & 4.5 & 48 \\
$200 \mathrm{GeV}$ & $0.16 \pm 0.11$ & $0.67 \pm 0.33$ & $4.3 \pm 3.7$ & 0.4 & 19 \\
$900 \mathrm{GeV}$ & $0.18 \pm 0.02$ & $0.72 \pm 0.11$ & $3.6 \pm 1.0$ & 6.1 & 50 \\
$2.76 \mathrm{TeV}$ & $0.19 \pm 0.02$ & $0.81 \pm 0.08$ & $3.4 \pm 0.4$ & 2.8 & 35 \\
$5.02 \mathrm{TeV}$ & $0.18 \pm 0.02$ & $0.80 \pm 0.08$ & $3.3 \pm 0.4$ & 3.2 & 35 \\
$7 \mathrm{TeV}$ & $0.18 \pm 0.02$ & $0.74 \pm 0.03$ & $3.1 \pm 0.1$ & 6.4 & 61 \\
$8 \mathrm{TeV}$ & $0.17 \pm 0.01$ & $0.74 \pm 0.06$ & $3.2 \pm 0.3$ & 3.3 & 40 \\
$13 \mathrm{TeV}$ & $0.18 \pm 0.02$ & $0.81 \pm 0.20$ & $3.2 \pm 1.5$ & 3.3 & 43 \\
\hline \hline
\end{tabular}

$\sqrt{s_{n n}}=7 \mathrm{TeV}$, in the range $|\eta|<0.5$ and $0.1<p_{\perp}<17.5$ $\mathrm{GeV} / c$. The obtained values of temperature are larger for charged particles than for pions, as expected.

In Table III, we show the fit results for $p \mathrm{~Pb}$ collisions at $\sqrt{s_{n n}}=5.02 \mathrm{TeV}$ in the range $-0.5<\eta<0$ and $0.1<p_{\perp}<$ $19 \mathrm{GeV} / c$. In Table IV, we show the fit results for XeXe collisions at $\sqrt{s_{n n}}=5.44 \mathrm{TeV}$ at different centralities in the range $\eta<0.8$ and $0.2<p_{\perp}<17 \mathrm{GeV} / c$, and in Table $\mathrm{V}$ for $\mathrm{PbPb}$ collisions at $\sqrt{s_{n n}}=5.02 \mathrm{TeV}$ at different centralities in the range $\eta<0.8$ and $0.2<p_{\perp}<17 \mathrm{GeV} / c$.

The general behavior of the temperatures $T_{h}$ and $T_{t h}$ in large systems is to increase with the colliding energy and centrality in all cases, as expected. The soft temperature $T_{t h}$ seems to be less sensitive to the collision energy in small systems. In $p p$ collisions, the minimum-bias distributions in Fig. 4 are well described under current uncertainties by a constant soft temperature around $T_{t h} \approx 0.15-0.20 \mathrm{GeV}$. The centrality dependence of this soft temperature $T_{t h}$ in small systems is different for light charged species than for kaons and unidentified charged particles. In $p p$ and $p \mathrm{~Pb}$ collisions, the soft temperature $T_{t h}$ of pions slightly decreases or can be considered constant with centrality, but it exhibits a clear enhancement with centrality for inclusive kaon and chargedparticle production. On the other hand, the behavior of the falloff index $k$ with centrality seems to be the same as the thermal temperature $T_{t h}$ : it increases with centrality except in light-particle production in $p p$ collisions, where it decreases

TABLE II. Characteristic temperatures and falloff $k$ of the TMD of $\pi^{ \pm}$in $p p$ collisions at $\sqrt{s_{n n}}=7 \mathrm{TeV}$ in the range $|\eta|<0.5$ and $0.1<p_{\perp}<17.5 \mathrm{GeV} / c$ [27] for several multiplicities.

\begin{tabular}{lccccc}
\hline \hline$d N_{c} / d \eta$ & $T_{t h}(\mathrm{GeV})$ & $T_{h}(\mathrm{GeV})$ & $k$ & $\chi^{2}$ & Ndf \\
\hline 21.3 & $0.14 \pm 0.01$ & $0.68 \pm 0.07$ & $3.1 \pm 0.5$ & 11.9 & 44 \\
16.5 & $0.14 \pm 0.01$ & $0.67 \pm 0.08$ & $3.1 \pm 0.7$ & 10.8 & 44 \\
13.5 & $0.14 \pm 0.01$ & $0.67 \pm 0.13$ & $3.1 \pm 1.2$ & 9.9 & 44 \\
11.5 & $0.14 \pm 0.01$ & $0.65 \pm 0.10$ & $3.1 \pm 0.8$ & 9.2 & 44 \\
10.1 & $0.14 \pm 0.01$ & $0.65 \pm 0.10$ & $3.1 \pm 0.9$ & 8.7 & 44 \\
8.45 & $0.14 \pm 0.01$ & $0.64 \pm 0.09$ & $3.1 \pm 0.8$ & 7.9 & 44 \\
6.72 & $0.14 \pm 0.01$ & $0.63 \pm 0.10$ & $3.2 \pm 0.9$ & 6.9 & 44 \\
5.4 & $0.14 \pm 0.01$ & $0.62 \pm 0.11$ & $3.2 \pm 1.0$ & 6.0 & 44 \\
3.9 & $0.15 \pm 0.01$ & $0.62 \pm 0.12$ & $3.3 \pm 1.2$ & 4.4 & 44 \\
2.3 & $0.15 \pm 0.01$ & $0.61 \pm 0.08$ & $3.4 \pm 0.5$ & 2.7 & 44 \\
\hline \hline
\end{tabular}

TABLE III. Characteristic temperatures and falloff $k$ of the TMD of $\pi^{ \pm}$in $p \mathrm{~Pb}$ collisions at $\sqrt{s_{n n}}=5.02 \mathrm{TeV}$ in the range $-0.5<\eta<$ 0 and $0.1<p_{\perp}<19 \mathrm{GeV} / c$ for several multiplicities [28].

\begin{tabular}{lccccc}
\hline \hline$d N_{c h} / d \eta$ & $T_{t h}(\mathrm{GeV})$ & $T_{h}(\mathrm{GeV})$ & $k$ & $\chi^{2}$ & Ndf \\
\hline 45.0 & $0.15 \pm 0.01$ & $0.72 \pm 0.03$ & $3.3 \pm 0.2$ & 12.7 & 54 \\
36.2 & $0.15 \pm 0.01$ & $0.72 \pm 0.08$ & $3.2 \pm 0.7$ & 11.8 & 54 \\
30.5 & $0.15 \pm 0.01$ & $0.71 \pm 0.03$ & $3.2 \pm 0.2$ & 11.2 & 54 \\
23.2 & $0.14 \pm 0.01$ & $0.70 \pm 0.05$ & $3.2 \pm 0.3$ & 10.0 & 54 \\
16.1 & $0.14 \pm 0.01$ & $0.68 \pm 0.03$ & $3.2 \pm 0.2$ & 8.9 & 54 \\
9.8 & $0.14 \pm 0.01$ & $0.65 \pm 0.08$ & $3.1 \pm 0.7$ & 7.8 & 54 \\
4.3 & $0.15 \pm 0.01$ & $0.62 \pm 0.09$ & $3.1 \pm 0.9$ & 5.8 & 54 \\
\hline \hline
\end{tabular}

or can be considered constant under current uncertainties. We then assume that, following the entanglement thermalization picture, a link exists between $T_{t h}$, a nonperturbative parameter, and the perturbative falloff $k$ of the hard processes, and we suggest the relation

$$
\frac{T_{h}}{k+1}=T_{t h},
$$

which approximately holds universally for all the analyzed energies, centralities, and colliding systems. In Fig. 4 we plot $T_{t h}$ and $T_{h} /(k+1)$ for $p p$ collisions at different energies. In Figs. 5-7 we plot the cases of $p p, p \mathrm{~Pb}, \mathrm{XeXe}$, and $\mathrm{PbPb}$ collisions at $\sqrt{s_{n n}}=7,5.02$, and $5.44 \mathrm{TeV}$ for different identified charged particles as a function of multiplicity. We have looked at the transverse momentum distribution of Higgs production decaying into $\gamma \gamma$ and $4 l$, as reported in Ref. [20], obtaining $T_{t h}=3.5 \pm 0.7 \mathrm{GeV}, T_{h}=14.4 \pm 0.3$, and $k=3.7 \pm 0.4$. Hence we obtain a quenched hard scale $T_{h} /(k+1)=3.1 \pm 0.4 \mathrm{GeV}$ to compare with $T_{t h}=3.5 \pm 0.7$ $\mathrm{GeV}$. In the same line, studies of the $p_{\perp}$ distribution of the $W \mathrm{~s}$ produced in $p \bar{p}$ collisions have found similar behavior $[33,34]$. An overall agreement is observed in all cases, except in very low multiplicity events and very low colliding energies in $p p$ collisions, where the discrepancies between both quantities becomes substantial. This agreement is remarkable considering the large number of TMDs studied and the large differences presented in the temperatures for different projectiles and targets, as well as centralities and energies.

To improve the agreement with $p p$ data, we devise also a convenient and simple parametrization of the whole soft and hard spectrum for the full range of energies explored at RHIC

TABLE IV. Characteristic temperatures and falloff $k$ of the TMD of charged particles in XeXe collisions at $\sqrt{s_{n n}}=5.44 \mathrm{TeV}$ for several centrality classes in the range $\eta<0.8$ and $0.2<p_{\perp}<$ $17 \mathrm{GeV} / c$, data taken from Ref. [31].

\begin{tabular}{lccccc}
\hline \hline$d N_{c h} / d \eta$ & $T_{t h}(\mathrm{GeV})$ & $T_{h}(\mathrm{GeV})$ & $k$ & $\chi^{2}$ & Ndf \\
\hline 1167 & $0.14 \pm 0.02$ & $0.68 \pm 0.08$ & $3.4 \pm 0.8$ & 27 & 48 \\
706 & $0.13 \pm 0.02$ & $0.68 \pm 0.03$ & $3.3 \pm 0.2$ & 17 & 48 \\
478 & $0.13 \pm 0.02$ & $0.66 \pm 0.03$ & $3.3 \pm 0.2$ & 12 & 48 \\
118 & $0.13 \pm 0.02$ & $0.64 \pm 0.10$ & $3.2 \pm 0.9$ & 3 & 48 \\
65 & $0.13 \pm 0.02$ & $0.63 \pm 0.30$ & $3.1 \pm 3.0$ & 5 & 48 \\
32 & $0.13 \pm 0.02$ & $0.65 \pm 0.13$ & $3.2 \pm 1.1$ & 5 & 48 \\
\hline \hline
\end{tabular}


TABLE V. Characteristic temperatures and falloff $k$ of the TMD of charged particles in $\mathrm{PbPb}$ collisions at $\sqrt{s_{n n}}=5.02 \mathrm{TeV}$ in the range $\eta<0.8$ and $0.2<p_{\perp}<17 \mathrm{GeV} / c$ for several centrality classes, data taken from Ref. [26].

\begin{tabular}{lccccc}
\hline \hline$d N_{c h} / d \eta$ & $T_{t h}(\mathrm{GeV})$ & $T_{h}(\mathrm{GeV})$ & $k$ & $\chi^{2}$ & Ndf \\
\hline 1942 & $0.14 \pm 0.02$ & $0.68 \pm 0.03$ & $3.5 \pm 0.2$ & 38 & 48 \\
1585 & $0.14 \pm 0.02$ & $0.68 \pm 0.03$ & $3.5 \pm 0.2$ & 32 & 48 \\
1180 & $0.13 \pm 0.02$ & $0.68 \pm 0.03$ & $3.4 \pm 0.2$ & 28 & 48 \\
786 & $0.13 \pm 0.02$ & $0.67 \pm 0.03$ & $3.3 \pm 0.2$ & 20 & 48 \\
512 & $0.13 \pm 0.02$ & $0.66 \pm 0.03$ & $3.3 \pm 0.2$ & 15 & 48 \\
318 & $0.13 \pm 0.02$ & $0.65 \pm 0.03$ & $3.2 \pm 0.2$ & 10 & 48 \\
183 & $0.13 \pm 0.02$ & $0.64 \pm 0.03$ & $3.2 \pm 0.2$ & 6 & 48 \\
96 & $0.13 \pm 0.02$ & $0.63 \pm 0.04$ & $3.2 \pm 0.2$ & 2 & 48 \\
45 & $0.13 \pm 0.01$ & $0.62 \pm 0.03$ & $3.1 \pm 0.1$ & 3 & 48 \\
\hline \hline
\end{tabular}

and the LHC. The hard part of the TMD (3) can be rewritten as

$$
\begin{aligned}
\frac{1}{\left(1+p_{\perp}^{2} / \gamma\right)^{k}} & =\int_{0}^{\infty} d x e^{-p_{\perp}^{2} x} \frac{\gamma}{\Gamma(k)}(\gamma x)^{k-1} e^{-\gamma x} \\
& :=\int_{0}^{\infty} d x e^{-p_{\perp}^{2} x} W_{p}(x, \gamma),
\end{aligned}
$$

with $\gamma \equiv k T_{h}^{2}$. Here, the function $e^{-p_{\perp}^{2} x}$ can be interpreted as the probability of production of a particle as a function of $p_{\perp}$ for a given occurrence of the hard momentum scale $x$, and then $W_{p}(x)$ is the distribution of these hard scales, in particular a Gamma distribution. Hence, $1 / k$ can be understood as the normalized fluctuations of the resulting hard scale $T_{h}$, since

$$
\frac{1}{k}=\frac{\left\langle x^{2}\right\rangle-\langle x\rangle^{2}}{\langle x\rangle^{2}},
$$

and $\langle x\rangle$ and $\langle x\rangle$ are momenta of $W_{p}(x)$. Next, in order to implement the quench relation and the emergence of the thermal temperature, we can add an additional source of fluctuations

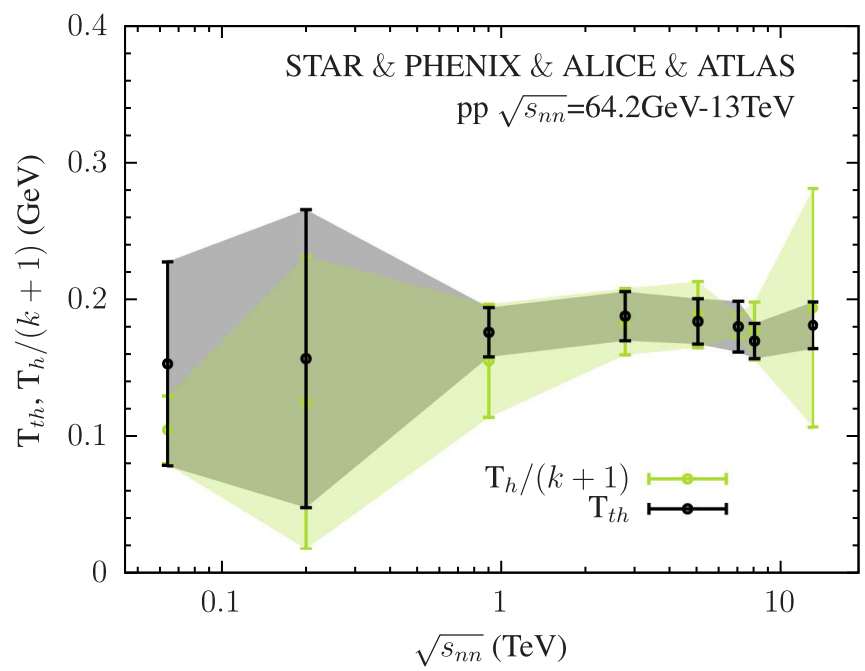

FIG. 4. Thermal scale $T_{t h}$ compared with the quenched hard scale $T_{h} /(k+1)$ of particles produced in $p p$ collisions at RHIC $[23,24]$ and LHC [25-29] as a function of collision energy.

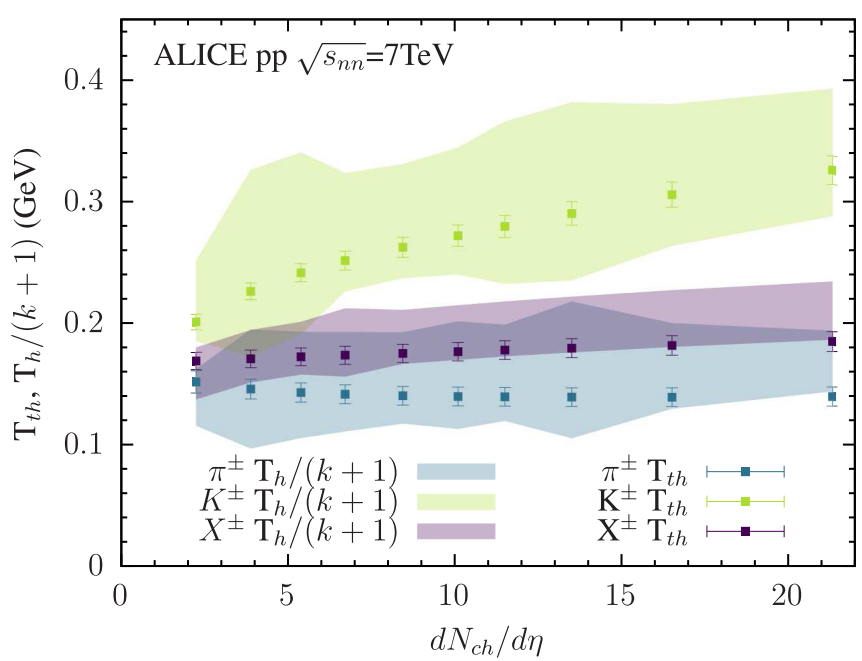

FIG. 5. Thermal scale $T_{t h}$ compared with the quenched hard scale $T_{h} /(k+1)$ extracted from ALICE collected data [27] on $p p$ collisions at $\sqrt{s_{n n}}=7 \mathrm{TeV}$, as a function of the charged particle multiplicity. Fits to (3) from $0.1<p_{\perp}<17.5 \mathrm{GeV} / c$ using $m_{\pi}=$ $0.14 \mathrm{GeV} / c^{2}, m_{K}=0.49 \mathrm{GeV} / c$, and $m_{X}=0.23 \mathrm{GeV} / c^{2}$, corresponding to the weighted averaged mass of the $\pi^{ \pm}, K^{ \pm}$, and $p$ channels.

for $T_{h}$ using a Gaussian distribution $G\left(T_{h}\right)$. The final TMD is then given by, using (5),

$$
\begin{aligned}
\frac{d N_{e v}^{2}}{d p_{\perp}^{2}}= & \int_{0}^{\infty} d T_{h} G\left(T_{h}\right) \frac{1}{\left(1+p_{\perp}^{2} / \gamma\right)^{k}} \\
= & \int_{0}^{\infty} d T_{h} G\left(T_{h}\right) \int_{0}^{\infty} d x W_{p}(x, \gamma) e^{-p_{\perp}^{2} x} \\
& :=\int_{0}^{\infty} d x W(x) e^{-p_{\perp}^{2} x},
\end{aligned}
$$

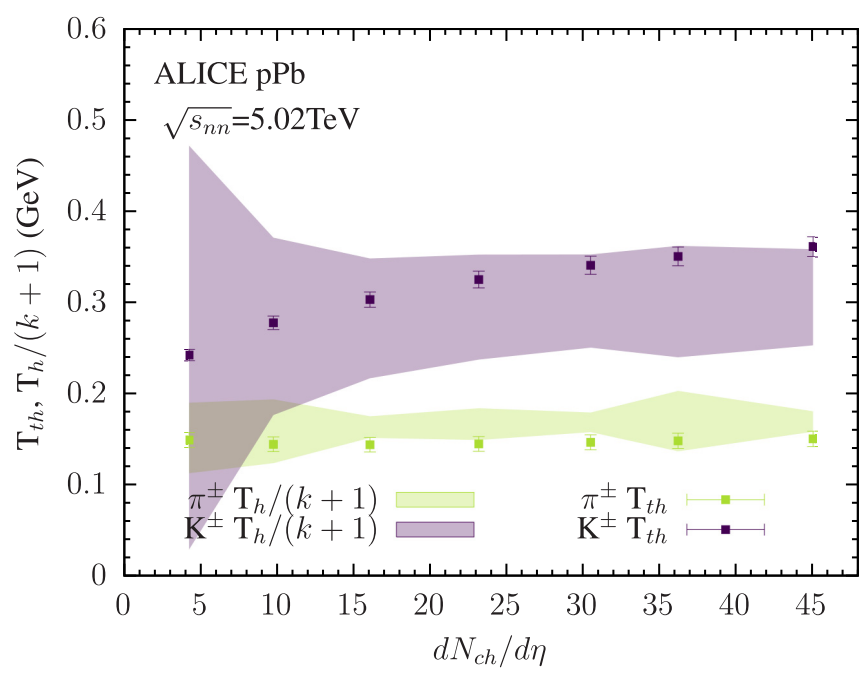

FIG. 6. Thermal scale $T_{t h}$ compared with the quenched hard scale $T_{h} /(k+1)$ of $\pi^{ \pm}$and $K^{ \pm}$extracted from ALICE collected data [30] on $p \mathrm{~Pb}$ collisions at $\sqrt{s_{n n}}=5.02 \mathrm{TeV}$ as a function of the chargedparticle multiplicity. Fit to (3) in the interval $0.1<p_{\perp}<19 \mathrm{GeV} / c$, with masses set to $m_{\pi}=0.14 \mathrm{GeV} / c^{2}$ and $m_{K}=0.49 \mathrm{GeV} / c^{2}$. 


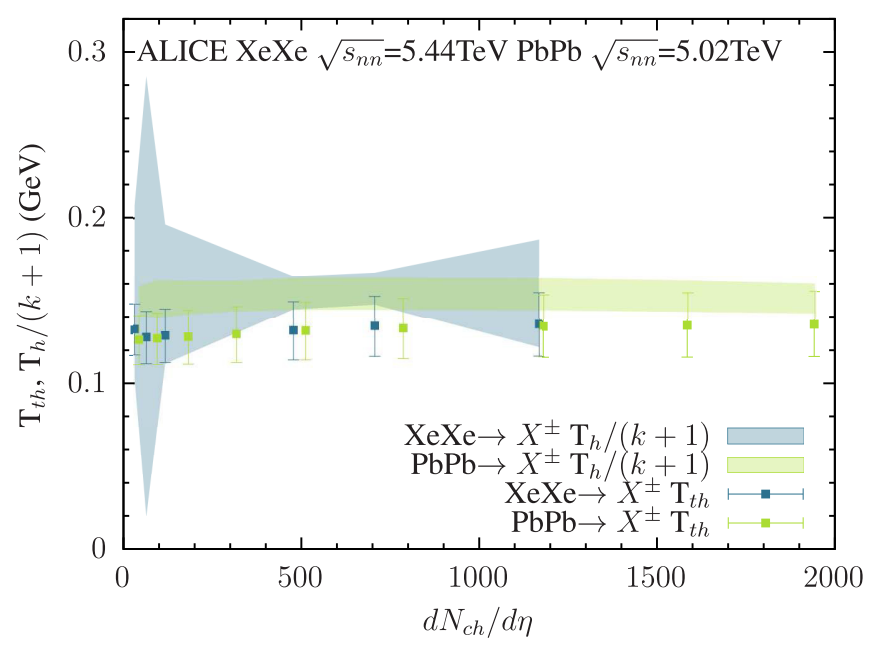

FIG. 7. Thermal scale $T_{t h}$ compared with the quenched hard scale $T_{h} /(k+1)$, extracted from a fit of (3) to ALICE collected data [26,31] on XeXe collisions at $\sqrt{s_{n n}}=5.44 \mathrm{TeV}$ in the interval $0.2<$ $p_{\perp}<17 \mathrm{GeV} / c$ and $\mathrm{PbPb}$ collisions at $\sqrt{s_{n n}}=5.02 \mathrm{TeV}$ in the interval $0.2<p_{\perp}<17 \mathrm{GeV} / c$, as a function of the charged-particle multiplicity. Unidentified charged particle mass set to $m_{X}=0.23$ $\mathrm{GeV} / c^{2}$, corresponding to the averaged weighted mass of the $\pi^{ \pm}$, $K^{ \pm}$, and $p$ channels.

where the fluctuation of the hard scale is encoded now in the new distribution $W(x)$, and it can be well approximated by

$$
W(x)=\int_{0}^{\infty} d T_{h} G\left(T_{h}\right) W_{p}(x, \gamma) \simeq \mathcal{N} \frac{x^{k-1}}{(1+x / \bar{x})^{k^{\prime}}},
$$

where $\mathcal{N}$ is a normalization constant, $\bar{x}$ is the scale of $x$, and $k^{\prime}=k+1 / 2$ is required to obtain Gaussian fluctuations at high $x$. The above equation has the correct asymptotic behavior for both low and high $x$. In this way the TMD in (7) becomes

$$
\frac{d N_{e v}^{2}}{d p_{\perp}^{2}}=\mathcal{N} \bar{x}^{k} \Gamma(k) U\left(k ; 1 / 2 ; \bar{x} p_{\perp}^{2}\right),
$$

where $U$ is the confluent hypergeometric function. The asymptotic limits for $p_{\perp} \rightarrow 0$ are

$$
\frac{d N_{e v}^{2}}{d p_{\perp}^{2}}=C\left(1-\frac{2 \Gamma(k+1 / 2)}{\Gamma(k)} \bar{x}^{1 / 2} p_{\perp}\right),
$$

and for $p_{\perp} \rightarrow \infty$,

$$
\frac{d N_{e v}^{2}}{d p_{\perp}^{2}}=\frac{C^{\prime}}{\left(\bar{x} p_{\perp}^{2}\right)^{k}} .
$$

At high $p_{\perp}$, a power-like behavior is obtained with power $2 k$, and at low $p_{\perp}$ the thermal behavior is $\exp \left(-p_{\perp} / T_{\mathrm{th}}\right)$ with

$$
T_{\mathrm{th}}=\frac{\Gamma(k)}{2 \Gamma(k+1 / 2)} \frac{1}{\bar{x}^{1 / 2}} .
$$

As the fluctuations encoded in the function $W(x)$ depend only on the scale $\bar{x}$ and $k$, we can say that the effective thermal temperature depends only on the scale $\bar{x}$ and its fluctuations. In Fig. 8, we show the fit using the whole soft and hard spectrum

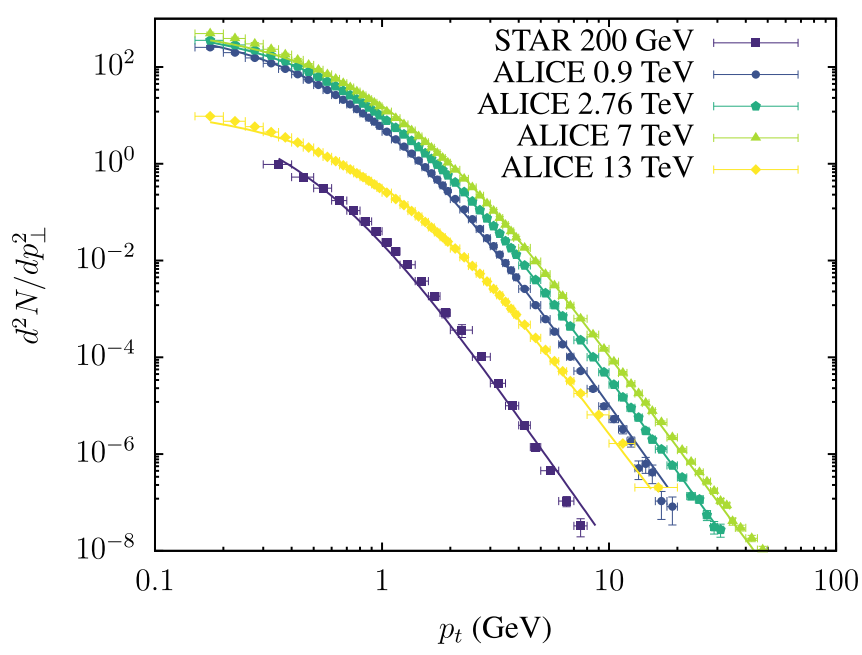

FIG. 8. TMD in (9) as a function of $p_{\perp}$ for $p p$ collisions at different energies (symbols, as marked) together with the fit to the hyper-geometric function (lines), using RHIC [24] and ALICE [25-28] data.

cast in the form (9) to $p p$ data at different energies. A good description is obtained in all cases.

\section{MULTIPLICITY DISTRIBUTION SCALES}

In what concerns to the multiplicity distribution, we note that a gamma distribution on the number of partons is also obtained for events which have at least one high- $p_{\perp}$ particle due to a hard parton collision. In fact, if $P(n)$ is the probability of having $n$ partons in a given collision, the probability $P^{c}(n)$ of having $n$ partons with at least one hard is $[35,36]$

$$
P^{c}(n)=\frac{n}{\langle n\rangle} P(n) .
$$

This selection procedure can be repeatedly applied, forming the chain

$$
P(n) \rightarrow \frac{n}{\langle n\rangle} P(n) \rightarrow \frac{n^{2}}{\left\langle n^{2}\right\rangle} P(n) \rightarrow \frac{n^{k}}{\left\langle n^{k}\right\rangle} P(n) .
$$

Similarly, we also notice that a gamma distributed multiplicity density convoluted with a Poisson process produces a negative binomial distribution (NBD) for the multiplicity, broadly used to describe the experimental data. Namely

$$
\begin{aligned}
& \frac{\Gamma\left(n+k_{n}\right)}{\Gamma(n+1) \Gamma\left(k_{n}\right)} \frac{\gamma_{n}^{k_{n}}}{\left(1+\gamma_{n}\right)^{k_{n}+n}} \\
& \quad=\int_{0}^{\infty} d N \frac{e^{-N} N^{n}}{n !}\left(\gamma_{n} N\right)^{k_{n}-1} \exp \left(-\gamma_{n} N\right) \\
& \quad=\int_{0}^{\infty} d N P(n, N) W_{n}(N),
\end{aligned}
$$

where as before

$$
\frac{1}{k_{n}}=\frac{\left\langle N^{2}\right\rangle-\langle N\rangle^{2}}{\langle N\rangle^{2}}, \quad \gamma_{n} \equiv \frac{k_{n}}{\langle N\rangle} .
$$


TABLE VI. Relation between the mean multiplicities for events having at least one hard parton and the mean multiplicity for the rest of the events extracted from Ref. [37].

\begin{tabular}{lcrc}
\hline \hline$\sqrt{s_{n n}}(\mathrm{TeV})$ & $\left\langle N_{s}\right\rangle$ & $\left\langle N_{h}\right\rangle$ & $\left\langle N_{h}\right\rangle /(k-1)$ \\
\hline 0.9 & $2.1 \pm 1.9$ & $5 \pm 4$ & $1.9 \pm 1.5$ \\
2.76 & $2.5 \pm 1.0$ & $7 \pm 2$ & $3.1 \pm 0.9$ \\
7 & $3.6 \pm 1.4$ & $12 \pm 3$ & $5.8 \pm 1.4$ \\
\hline \hline
\end{tabular}

Since the mean multiplicity and the $p_{\perp}$ distribution are related as follows:

$$
\langle n\rangle=\int d^{2} p_{\perp} \int d x f\left(p_{\perp}, x\right) W_{p}(x),
$$

the following relation between the two gamma distributions can be written:

$$
W_{p}(x)=\frac{\gamma_{n}}{\gamma} W_{n}(x)
$$

with $k=k_{n}+2$. The convolution of the gamma distribution with a Poisson distribution gives rise to a negative binomial distribution for the multiplicity distribution, see formula (15). As far as the gamma distribution is obtained for at least one hard parton, the resulting multiplicity distribution describes the multiplicity distribution events with at least one hard parton. For the rest of events another distribution is required. There are several fits to the $p p$ data at different colliding energies using two negative binomial distributions [37-40]. Each of these distributions has two parameters, $k$ fixing the fluctuations and $\langle n\rangle$ the mean multiplicity. The two mean multiplicities can be seen as the two multiplicity scales corresponding to the two transverse momentum scales $T_{t h}$ and $T_{h}$, and we can look for a relation between these scales similar to the relation (4).

According to formula (18), the parameter $k$ of the gamma distribution is two units larger than the one corresponding to the gamma distributions on the number of partons, thus we expect that instead of the equation (4) for the $p_{\perp}$ distribution, we should have

$$
\frac{\left\langle N_{h}\right\rangle}{k-1}=\left\langle N_{s}\right\rangle .
$$

This equation must be seen with caution because in the case of multiplicities longitudinal momentum fluctuations add to the $p_{\perp}$ fluctuations. To avoid these contributions we look at the data on small rapidity range. In Table VI we show the results of a fit [37] using two negative binomial distributions to the multiplicity distributions of $p p$ collisions in the pseudorapidity range $|\eta| \leqslant 0.5$ for different energies. The comparison of columns two and four shows a reasonable agreement.

\section{DISCUSSION}

In the left-hand side of equation (4), $T_{h}$ and $k$ are parameters related to hard collisions and thus described by perturbative QCD. On the other hand $T_{t h}$ has to do with nonperturbative QCD. Thus, to some extent, equation (4) links perturbative and nonperturbative physics. The factor $k+1$ in equation (4) determines the falloff of the probability of having an additional hard parton normalized by the hard scale $T_{h}^{2}$. Such probability is just the variation of the hard transverse momentum distribution.

In this thermalization scenario implemented by the nucleon wave-function collapse in the hard process, the exact form of the relation between the soft and hard sectors is nevertheless unknown. Hence the proposed relation (4) has to be understood as a phenomenological finding satisfying the qualitative features of this rapid quench picture. In addition, the relation for the hard and soft scales seems to be consistent within the energy and the multiplicity distributions. We notice that Eq. (4) may hold also for a thermalization procedure in which the equilibrium temperature $T_{t h}$ is proportional to the number of hard events or collisions, encoded in the quotient $T_{h} /(k+1)$. That is, the number of hard events in the inclusive cross section is proportional to the width of the distribution $T_{h}$ and inversely proportional to the falloff $k$.

Relations between these two regimes have been recently put forward in different quantum problems [41] and, more recently, have been suggested as entropy constraints in an entangled nucleon, relating the final-state multiplicity of the fragmenting nucleon with the parton distribution function probed by hard processes in $p p$ and $e p$ collisions [7]. Testing the implications of this entanglement, the $\mathrm{H} 1$ collaboration has measured very recently the charged particle distribution in DIS at HERA [42]. The hadron entropy found in data does not confirm, however, these entanglement predictions.

It is convenient to discuss if this disagreement is related to the way in which the entanglement entropy of the nucleon has to be obtained. There is not, to our knowledge, a known way of computing from first principles the distribution of weights in the entangled nucleon. Cascade models, not including saturation and nonlinear evolution [19], may not be sensitive enough to correctly describe the initial entanglement entropy. Thus the observed entropy in the multiplicity distribution of hadrons may not be in an one-to-one correspondence with the entropy of the cascade.

However, we notice that the observed multiplicity distributions in data are well described by NBDs. We can devise then a way of reconstructing the cascade process under these phenomenological considerations. We may assume the weights of the entangled state to be formed according to a Poisson process, with a given mean value $\lambda$. Then each of these partons gives rise to a cascade with a geometric distribution [19], with parameter $p=\langle n\rangle /\left(\langle n\rangle+k_{n}\right)\left(k_{n}=1\right.$ for a geometric distribution). For the case of DIS at the energies explored by the $\mathrm{H} 1$ collaboration [42], the falloff parameter $k_{n}$ is large enough to transform back the final NBD to the initial Poisson distributed multiplicity. Following this observation, $p$ has to be small and the final-state multiplicity mirrors the initial weight distribution instead of being geometrically distributed. At larger energies, however, $p$ must increase so that the part of the cascade becomes more important.

At the level of the von Neumann entropy, this energy evolution corresponds to the passage from a Poisson distributed entropy to a geometric or Gamma

$$
\ln \langle n\rangle^{1 / 2} \rightarrow \ln \langle n\rangle
$$


the falloff $k$ of the NBD interpolating between these two limits [21]. At LHC energies for $p p$ collisions we expect that the parameter $p$ is large enough that the entropy is dominated by the geometric term and $S \propto \ln \langle n\rangle$, as was pointed out in Ref. [7]. At even larger energies due to saturation of partons we expect to recover a behavior $S \propto \ln \langle n\rangle^{1 / 2}$. Accordingly, the number of microstates is no longer $n$ but saturates as $\sqrt{n}$ when the colliding energy increases, following the expectations of the glasma picture of the CGC [43] or the string percolation model [44]. Recently, a re-analysis of the H1 collaboration data [45] with the adequate corrections for the different kinematical regions has produced agreement with the entanglement predictions. These corrections are in line with the above discussion.

\section{CONCLUSIONS}

Summarizing, the analysis of the transverse momentum distributions of $p p, p \mathrm{~Pb}, \mathrm{XeXe}$, and $\mathrm{PbPb}$ collisions at different RHIC and LHC energies and centralities together with Higgs production decaying into $\gamma \gamma$ and $4 l$ suggest that a hard collision provides an ultraviolet scale that quenches the spectrum by means of fluctuations of the hard scale. A simple relation between the effective temperature and the hard scales is obtained which is approximately satisfied in the different cases in study. In this way, a nonperturbative scale $T_{t h}$ has been related to two perturbative quantities, $T_{h}$ and $k$. A Gamma distribution is found, in agreement with phenomenological descriptions, for the distribution of the hard scale as well as the number of partons. The normalized fluctuations of both distributions are related and give rise to a relation between the multiplicities of the soft and hard spectrum. These findings are in line with the possibility that a hard parton collision works as an ultraviolet cutoff producing a quench of the rest of the entanglement partons of the initial wave function. Such entanglement may be at the origin of the apparent thermalization of the colliding hadrons.

\section{ACKNOWLEDGMENTS}

We thank N. Armesto for a critical reading of the paper. We thank the grant María de Maeztu Unit of Excellence under project MDM-2016-0692 of Ministry of Science and Innovation of Spain. This work has been funded by Ministerio Ciencia e Innovación of Spain under projects FPA201783814-P and Xunta de Galicia (Spain) (Centro singular de investigación de Galicia accreditation 2019-2022) by European Union ERDF, and by the "María de Maeztu" Units of Excellence program MDM-2016-0692. X.F. is supported by grant ED481B-2019-040 (Xunta de Galicia) and the Fulbright Visiting Scholar fellowship.
[1] L. Yi, Study of small colliding systems, Nucl. Phys. A 982, 85 (2019).

[2] M. Strickland, Small system studies: A theory overview, Nucl. Phys. A 982, 92 (2019).

[3] P. Calabrese and J. Cardy, Evolution of entanglement entropy in one dimensional systems, J. Stat. Mech.: Theory Exp. (2005) 04010.

[4] P. Calabrese and J. Cardy, Quantum quenches in $1+1$ dimensional conformal field theories, J. Stat. Mech.: Theory Exp. (2016) 064003.

[5] J. Bergers, S. Floerchinger, and R. Venugopalan, Dynamics of entanglement in expanding quantum fields, J. High Energy Phys. 04 (2018)145.

[6] J. Bergers, S. Floerchinger, and R. Venugopalan, Thermal excitation spectrum from entanglement in an expanding quantum string, Phys. Lett. B 778, 442 (2018).

[7] Z. Tu, D. E. Kharzeev, and T. Ullrich, Einstein-Podolsky-Rosen Paradox and Quantum Entanglement at Subnucleonic Scales, Phys. Rev. Lett. 124, 062001 (2020).

[8] A. Kovner, M. Lublinsky, and M. Serino, Entanglement entropy, entropy production and time evolution in high energy QCD, Phys. Lett. B 792, 4 (2019).

[9] N. Armesto, F. Domínguez, A. Kovner et al., The Color Glass Condensate density matrix Lindblad evolution, entanglement entropy and Wigner functional, J. High Energy Phys. 05 (2019) 025 .

[10] E. Gotsman and E. Levin, High energy QCD: Multiplicity distribution and entanglement entropy, Phys. Rev. D 102, 074008 (2020).

[11] E. Gotsman and E. Levin, Thermal radiation and inclusive production in the Kharzeev-Levin-Nardi model for ion-ion collisions, Phys. Rev. D 100, 034013 (2019).
[12] E. Gotsman and E. Levin, Thermal radiation and inclusive production in the $\mathrm{CGC} /$ saturation approach at high energies, Eur. Phys. J. C 79, 415 (2019).

[13] A. V. Giannini, V. P. Goncalves, and P. V. R. G. Silva, Thermal radiation and inclusive production in the running coupling $k_{T}$ factorization approach, Eur. Phys. J. A 57, 43 (2021).

[14] G. Iskander, J. Pan, M. Tyler, C. Weber, and O. K. Baker, Quantum entanglement and thermal behavior in charged-current weak interactions, Phys. Lett. B 811, 135948 (2020).

[15] P. Castorina, A. Iorio, D. Lanteri, and P. Lukes, Gluon shadowing and nuclear entanglement, Int. J. Mod. Phys. E 30, 2150010 (2021)

[16] Y. Afik and J. R. M. de Nova, Entanglement and quantum tomography with top quarks at the LHC, Eur. Phys. J. Plus 136, 907 (2021).

[17] G. S. Ramos and M. V. T. Machado, Investigating entanglement entropy at small $x$ in DIS off protons and nuclei, Phys. Rev. D 101, 074040 (2020).

[18] A. Baty, P. Gardner, and W. Li, Collective evolution of a parton in the vacuum: the ultimate partonic "droplet", non-perturbative QCD and quantum entanglement, arXiv:2104.11735.

[19] D. E. Kharzeev and E. Levin, Deep inelastic scattering as a probe of entanglement, Phys. Rev. D 95, 114008 (2017).

[20] O. K. Baker and D. E. Kharzeev, Thermal radiation and entanglement in $p$ - $p$ collisions at the LHC, Phys. Rev. D 98, 054007 (2018).

[21] X. Feal, C. Pajares, and R. Vazquez, Thermal behavior and entanglement in $\mathrm{PbPb}$ and $p p$ collisions, Phys. Rev. C 99, 015205 (2019).

[22] R. Bellwied, Quantum entanglement in the initial and final state in relativistic heavy ion collisions, J. Phys.: Conf. Ser. 1070, 012001 (2018). 
[23] A. Adare et al. (PHENIX Collaboration), Inclusive cross section and double helicity asymmetry for $\pi^{0}$ production in $p p$ collisions at $\sqrt{s_{N N}}=62.4 \mathrm{GeV}$, Phys. Rev. D 79, 012003 (2009).

[24] J. Adams et al. (STAR Collaboration), Identified hadron spectra at large transverse momentum in $p+p$ and $d+\mathrm{Au}$ collisions at $\sqrt{s_{N N}}=200 \mathrm{GeV}$, Phys. Lett. B 637, 161 (2006).

[25] B. Abelev et al. (ALICE Collaboration), Energy dependence of the transverse momentum distributions of charged particles in $p p$ collisions measured by ALICE, Eur. Phys. J. C 73, 2662 (2013).

[26] S. Acharya et al. (ALICE Collaboration), Transverse momentum spectra and nuclear modification factors of charged particles in $p p, p-\mathrm{Pb}$ and $\mathrm{Pb}-\mathrm{Pb}$ collisions at the LHC, J. High Energy Phys. 11 (2018) 013.

[27] S. Acharya et al. (ALICE Collaboration), Multiplicity dependence of light-flavor hadron production in $p p$ collisions at $\sqrt{s_{N N}}=7$ TeV, Phys. Rev. C 99, 024906 (2019).

[28] J. Adam et al. (ALICE Collaboration), Pseudorapidity and transverse-momentum distributions of charged particles in proton-proton collisions at $\sqrt{s_{N N}}=13 \mathrm{TeV}$, Phys. Lett. B 753, 319 (2016).

[29] G. Aad et al. (ATLAS Collaboration), Charged-particle distributions in $p p$ interactions at $\sqrt{s}=8 \mathrm{TeV}$ measured with the ATLAS detector, Eur. Phys. J. C 76, 403 (2016).

[30] A. Jaroslav et al. (ALICE Collaboration), Multiplicity dependence of charged pion, kaon and (anti)proton production at large transverse momentum in $p$ - $\mathrm{Pb}$ collisions at $\sqrt{s_{N N}}=5.02 \mathrm{TeV}$, Phys. Lett. B 760, 720 (2016).

[31] ALICE Collaboration, Transverse momentum spectra and nuclear modification factors of charged particles in $\mathrm{Xe}-\mathrm{Xe}$ collisions at $\sqrt{s_{N N}}=5.44 \mathrm{TeV}$, Phys. Lett. B 788, 166 (2019).

[32] A. A. Bylinkin, D. E. Kharzeev, and A. A. Rostovtsev, The origin of thermal component in the transverse momentum spectra in high energy hadronic processes, Int. J. Mod. Phys. E 23, 1450083 (2014).
[33] C. Albajar et al. (UA1 Collaboration), Studies of intermediate vector boson production and decay in UA1 at the CERN protonantiproton collider, Z. Phys. C: Part. Fields 44, 15 (1989).

[34] R. S. Fletcher, F. Halzen, A. Grau, G. Pancheri, and Y. N. Srivastava, Do we understand the resummation of soft gluons in $W$ events? Phys. Lett. B 237, 113 (1990).

[35] J. Dias de Deus, C. Pajares, and C. A. Salgado, Production associated to rare events in high energy hadron-hadron collisions, Phys. Lett. B 408, 417 (1997).

[36] J. Dias de Deus, C. Pajares, and C. A. Salgado, Multiplicity and transverse energy distributions associated to rare events in nucleus-nucleus collisions, Phys. Lett. B 409, 474 (1997).

[37] A. Alkin, Phenomenology of charged-particle multiplicity distributions, Ukr. J. Phys. 62, 743 (2017).

[38] ALICE Collaboration, Charged particle multiplicity distribution over a wide range of multiplicities in proton-proton collisions, Eur. Phys. J. C 77, 852 (2017).

[39] I. Zborovský, Three-component multiplicity distribution, oscillation of combinants and properties of clans in $p p$ collisions at the LHC, Eur. Phys. J. C 78, 816 (2018).

[40] M. Biyajima and T. Mizoguchi, Unified description of multiplicity distributions and Bose-Einstein correlations at the LHC based on the three-negative binomial distribution, Int. J. Mod. Phys. A 34, 1950203 (2019).

[41] O. Costin and G. V. Dunne, Physical resurgent extrapolation, Phys. Lett. B 808, 135627 (2020).

[42] H1 Collaboration, Measurement of charged particle multiplicity distributions in DIS at HERA and its implication to entanglement entropy of partons, arXiv:2011.01812.

[43] L. D. McLerran and R. Venugopalan, Computing quark and gluon distribution functions for very large nuclei, Phys. Rev. D 49, 2233 (1994).

[44] J. Dias de Deus and C. Pajares, String percolation and the glasma, Phys. Lett. B 695, 211 (2011).

[45] D. E. Kharzeev and E. Levin, Deep inelastic scattering as a probe of entanglement confronting experimental data, Phys. Rev. D 104, L031503 (2021). 